\title{
hnRNPK modulates selective quality-control autophagy by downregulating the expression of HDAC6 in 293 cells
}

\author{
ZHIPENG LI* ${ }^{*}$ XIAOHUI LIU* ${ }^{*}$ JIE MA, TINGTING ZHANG, XUEJUAN GAO and LANGXIA LIU \\ Key Laboratory of Functional Protein Research of Guangdong Higher Education Institute, \\ Institute of Life and Health Engineering, College of Life Science and Technology, \\ Jinan University, Guangzhou, Guangdong 510632, P.R. China
}

Received March 9, 2018; Accepted June 22, 2018

DOI: $10.3892 /$ ijo.2018.4517

\begin{abstract}
RNPK modulates selective quality-control autophagy bAbstract. A recent study has reported that heterogeneous nuclear ribonucleoprotein K (hnRNPK) regulated autophagy in leukemia cells. However, the underlying mechanism of this remains elusive. The present study assessed the role of hnRNPK in the autophagy of the 293 cell line and investigated the associated molecular mechanisms of this. It was revealed that hnRNPK-knockdown with siRNA or the CRISPR-Cas9 system could increase the level of autophagy, while hnRNPK-overexpression exerted the opposite effect in 293 cells under normal nutrient conditions. By contrast, hnRNPK-knockdown or -overexpression had no effect on serum starvation- or rapamycin-induced autophagy. Therefore, hnRNPK was likely involved in the late stage of autophagy rather than the early stage. Furthermore, it was observed that hnRNPK deficiency led to the decrease in $\alpha$-tubulin K40 acetylation in hnRNPK single allele knockout (hnRNPK $\left.{ }^{+/}\right)$cells. In accordance with this result, it was revealed that the mRNA and protein levels of histone deacetylas 6 (HDAC6) were upregulated in hnRNPK ${ }^{+/-}$cells compared with the wild-type cells. As a consequence, autophagosome-lysosome fusion in hnRNPK ${ }^{+-}$ cells was significantly enhanced and could be effectively suppressed by treatment with the selective inhibitor of HDAC6, tubastatin A (Tub A). Furthermore, prominent co-localization of ubiquitin-positive aggregates with LC3-positive autophagosomes was observed in hnRNPK ${ }^{+-}$cells, but not in the wild-type
\end{abstract}

Correspondence to: Professor Langxia Liu or Ms Xiaohui Liu, Key Laboratory of Functional Protein Research of Guangdong Higher Education Institute, Institute of Life and Health Engineering, College of Life Science and Technology, Jinan University, Guangzhou, Guangdong 510632, P.R. China

E-mail: langxialiu@gmail.com

E-mail: 41304912@qq.com

${ }^{*}$ Contributed equally

Key words: heterogeneous nuclear ribonucleoprotein $\mathrm{K}$, qualitycontrol, autophagy, histone deacetylase 6, autophagosome-lysosome fusion or hnRNPK ${ }^{+/}$cells treated with Tub A. Taken together, these results suggested that hnRNPK may regulate basal autophagy through modulating the expression level of HDAC6 to influence the autophagosome-lysosome fusion.

\section{Introduction}

Autophagy serves roles in a variety of physiological and pathophysiological processes, including starvation response, intracellular protein and organelle clearance, development, anti-aging, elimination of microorganisms, cell death, tumor suppression and antigen presentation (1). Dysfunction of autophagy is associated with cancer, neurodegeneration, microbial infection and aging (2). Autophagy has long been characterized as a non-selective degradative pathway activated by starvation. However, it has become evident and important that autophagy enforces intracellular qualitycontrol (QC) by selective disposal of damaged proteins and organelles for maintaining normal cellular homeostasis, and this nutrient-independent selective autophagy occurs at basal, constitutive levels $(3,4)$. It is now generally accepted that selective autophagy receptors, including mammalian p62/SQSTM1, determine exquisite target specificity $(5,6)$. p62 has LC3- and ubiquitin-binding domains, allowing it to mediate selective recognition of polyubiquitinated protein aggregates. The mechanism of selective autophagosome formation consists of a series of molecular events beginning with the modification of the cargo by ubiquitination, followed by its recognition by the polymeric autophagy receptors, including p62, contributing toward its further modification and the recruitment of specific autophagy-related (ATG) proteins, leading to the fusion of the completed autophagosome with a lysosome, and degradation of the cargo and receptor (6). Autophagy is a complex process that requires a tight coordination among distinct molecular systems $(7,8)$. In addition to the core autophagy-related proteins, the secretory and endocytic pathways, and the cytoskeletal network also serve important roles during autophagy, including facilitating autophagosome transport and enabling clearance of autophagic substrates (9).

Heterogeneous nuclear ribonucleoprotein K (hnRNPK) is a DNA/RNA binding protein shuttling between the cell nucleus and cytoplasm. Its functions are mainly associated with the regulation of gene expression by controlling the RNA 
metabolism, including the synthesis, splicing, nuclear export, translation and decay of various mRNAs (10-13). Recent studies have also demonstrated that hnRNPK may regulate multiple cell signaling pathways by physical or/and functional interactions with various components of these pathways, including PKC, Src, ERK and GSK-3 $\beta$ kinases (14-21). Notably, abnormal expression of hnRNPK has been observed in various types of cancer, including non-small cell lung cancer (22), colorectal cancer (23), prostate cancer (24), squamous cell carcinoma (25) and nasopharyngeal carcinoma (26). An increasing volume of evidence has suggested that hnRNPK serves critical roles in numerous cellular processes, including cell proliferation, differentiation, apoptosis and motility $(27,28)$. Notable, Zhang et al (29) recently demonstrated that hnRNPK contributed toward drug resistance in acute myeloid leukemia through the regulation of autophagy (29). However, the mechanism by which hnRNPK modulates autophagy remains to be elucidated.

Our previous study demonstrated that hnRNPK-knockdown could significantly decrease the acetylation of $\alpha$-tubulin on Lys40 in RAW264.7 cells, which may influence the microtubule stability and thereby regulate the formation and dynamic patterning of podosomes (30). Recent reports have highlighted the contribution of acetylation toward autophagy control and revealed that acetylated microtubules are essential for the fusion of autophagosomes with lysosomes $(31,32)$. $\alpha$-tubulin is specifically acetylated on Lys40 (K40), which is mainly catalyzed by a conserved $\alpha$-tubulin acetyltransferase ( $\alpha$-TAT) (33). The X-ray crystal structure of human $\alpha$-TAT and the chemical basis for $\alpha$-tubulin K40 acetylation, together with the catalytic mechanism of $\alpha$-TAT elucidated through a detailed enzymatic analysis has indicated that $\alpha$-TAT selectively acetylates $\alpha$-tubulin K40 (34,35). Hubbert et al (36) reported that HDAC6 functions as a tubulin deacetylase. This aforementioned study indicated that overexpression of HDAC6 leads toward a global deacetylation of $\alpha$-tubulin, whereas a decrease in the expression levels of HDAC6 increased $\alpha$-tubulin acetylation in vivo. Furthermore, another previous study demonstrated that recombinant HDAC6 protein potently deacetylates $\alpha$-tubulin in assembled microtubules in vitro. These convergent results prompted the aim of the present study to investigate whether hnRNPK regulates autophagy by modulation of $\alpha$-tubulin acetylation and to investigate the related regulatory mechanisms.

The present study used the 293 cell line as a cellular model to study the role of hnRNPK in basal or induced autophagy. It was revealed that hnRNPK modulated selective quality-control autophagy but was dispensable for non-selective, starvationor rapamycin-induced autophagy. hnRNPK overexpression partially reversed chloroquine (CQ)-inhibited autophagy, but had no effect on the 3-methyladenine (3-MA)-mediated inhibition of autophagy, suggesting its role at the late stage of autophagy instead of the early stage. Using a stable hnRNPK single allele knockout (KO) (hnRNPK $\left.{ }^{+-}\right) 293$ cell line established using the CASPR-Cas 9 system, it was demonstrated that hnRNPK deficiency led to an increase in HDAC6 mRNA and protein levels, together with a decrease in $\alpha$-tubulin K40 acetylation. A mCherry-GFP-LC3 reporter assay demonstrated that autophagosome-lysosome fusion in hnRNPK ${ }^{+/-}$cells was significantly enhanced in these cells compared with the wildtype cells, which could be effectively suppressed by treatment with tubastatin A (Tub A), a selective inhibitor of HDAC6.
In addition, a prominent co-localization of ubiquitin-positive aggregates with LC3-positive autophagosomes was observed in hnRNPK ${ }^{+/-}$cells, but not in the wild-type group or hnRNPK ${ }^{+-}$ cells treated with Tub A. Taken together, these results suggested that hnRNPK may serve a pivotal role in the basal autophagy by transcriptionally regulating the expression levels of HDAC6 to influence autophagosome-lysosome fusion.

\section{Materials and methods}

Cell lines and reagents. The 293 cell line (Cell Resource Center, Institute of Life Science Chinese Academy of Sciences, Shanghai, China) was cultured in Dulbecco's modified Eagle's medium (DMEM; Gibco; Thermo Fisher Scientific, Inc., Waltham, MA, USA) supplemented with $10 \%$ fetal bovine serum (FBS; GE Healthcare, Chicago, IL, USA) at $37^{\circ} \mathrm{C}$ in a humidified atmosphere containing $5 \% \mathrm{CO}_{2} .293$ cells at a confluence of $80-90 \%$ were transfected using Lipofectamine 3000 reagent (Invitrogen; Thermo Fisher Scientific, Inc.). Rapamycin, 3-methyladenine, MG132, Tubulin-Tracker Red and SDS lysis buffer were purchased from Beyotime Institute of Biotechnology (Haimen, China). Tubastatin A HCl was purchased from SigmaAldrich; Merck KGaA (Darmstadt, Germany). Chloroquine was purchased from Selleck Chemicals (Houston, TX, USA). The mCherry-GFP-LC3B plasmid and pCMV-flag-hnRNPK plasmids were constructed in our laboratory. Primary antibodies against the following proteins were used: hnRNPK (cat. no. sc-28380; Santa Cruz Biotechnology, Inc., Dallas, TX, USA), LC-3B (cat. no. 2775S; CST Biological Reagents Co., Ltd., Shanghai, China), HDAC6 (cat. no. 12834-1-AP; ProteinTech Group, Inc., Chicago, IL, USA), p62/SQSTM1 (cat. no. 661841-AP; ProteinTech Group, Inc.), $\alpha$-TAT1 (cat. no. ab58742; Abcam, Cambridge, MA, USA), $\alpha$-tubulin (cat. no. 11224-1AP; ProteinTech Group, Inc.), $\beta$-tubulin (cat. no. 10094-1-AP; ProteinTech Group, Inc.), acetylated $\alpha$-tubulin (cat. no. T7451; Sigma-Aldrich; Merck KGaA), ubiquitin (cat. no. ab134953; Abcam, Inc.) and GAPDH (cat. no. ZS-25778, OriGene Technologies, Inc., Beijing, China). The antibodies were diluted in Tris-buffered saline with Tween-20 buffer (dilution, 1:2,000; cat. no. 9997; CST Biological Reagents Co., Ltd.) containing 5\% dry skimmed milk.

CRISPR/Cas9-mediated hnRNPK KO in 293 cells. The CRISPR/Cas9 plasmid was purchased from Genloci Biotechnologies, Inc. (Jiangsu, China). The sequences of gRNA were as follows: Strand A, 5'-caccGATGATGTTTGAT GACCGTCG-3' and strand B, 5'-aaacCGACGGTCATCAAAC ATCATC-3'. The sequences of non-targeting gRNA (negative control) were as follows: Strand A, 5'-caccGGGTCTTCGAGAAGACCT-3' and strand B, 5'-aaacAGGTCTTCTCGA AGACCC-3'. These gRNAs were synthesized by Shanghai GenePharma Co., Ltd. (Shanghai, China). According to the manufacturer's protocol, transfection was performed with CRISPR/Cas9 expressing plasmid carrying hnRNPK gRNA in a 6 -well plate with a seeding density of $3 \times 10^{6}$ cells/well. A mixture of $2.5 \mu \mathrm{g} /$ well DNA and Lipofectamine 3000 was added, followed by incubation for $48 \mathrm{~h}$. A total of $2 \mu \mathrm{g} / \mathrm{ml}$ puromycin was used for the selection. The established 293 hnRNPK-knockdown cell line was cultured in DMEM with $10 \%$ FBS and $2 \mu \mathrm{g} / \mathrm{ml}$ puromycin. The medium was 
changed twice per week. Isolation of cell colony populations from the selected cells was performed by serial dilutions and cells with a single allele $\mathrm{KO}$ were harvested.

hnRNPK siRNA transfection. 293 cells were transfected in suspension with $100 \mathrm{nM}$ hnRNPK siRNA or non-targeting siRNA. Briefly, $2.0 \times 10^{5}$ cells in a $200 \mu$ l suspension were mixed with $200 \mu \mathrm{l}$ opti-MEM plus and $5 \mu \mathrm{l}$ Lipofectamine 2000 , and incubated at room temperature for $10 \mathrm{~min}$, prior to being seeded into a 6-well plate with $200 \mu 1$ complete DMEM supplemented with $10 \%$ FBS. For an effective hnRNPKknockdown, a mixture of two siRNAs with a final concentration of $100 \mathrm{nM}$ was used, including the following sequences: hnRNPK siRNA-1 forward, 5'-UAUUAAGGCUCUCCGUA CATT-3' and reverse, 5'-UGUACGGAGAGCCUUAAU ATT-3'; hnRNPK-siRNA-2 forward, 5'-CCUUAUGAUCCC AACUUUUTT-3' and reverse, 5'-AAAAGUUGGGAUCAU AAGGTT-3'; negative control siRNA (NC): forward, 5'-UUC UCCGAACGUGUCACGUTT-3' and reverse, 5'-ACGUGAC ACGUUCGGAGAATT-3'. The treatment was performed $24 \mathrm{~h}$ after transfection and the siRNAs were synthetized by Shanghai GenePharma Co., Ltd.

Detection of protein expression by western blotting. Cells were harvested and lysed with a lysis buffer [ $20 \mathrm{mM}$ Tris (pH 7.5), $150 \mathrm{mM} \mathrm{NaCl}, 1 \%$ Triton X-100, sodium pyrophosphate, $\beta$-glycerophosphate, EDTA, $\mathrm{Na}_{3} \mathrm{VO}_{4}$, leupeptin and $1 \%$ protease inhibitor cocktail (Roche Applied Science, Penzberg, Germany)]. Protein concentrations were measured using a bicinchoninic acid protein assay (Pierce; Thermo Fisher Scientific, Inc.). A total of $50 \mu \mathrm{g}$ protein were separated by 10 or $12 \%$ SDS-PAGE and transferred to polyvinylidene difluoride membranes (EMD Millipore, Billerica, MA, USA). Following blocking in 5\% dry skimmed milk for $60 \mathrm{~min}$ at room temperature, membranes were incubated with the primary antibodies (dilution, 1:2,000) overnight at $4^{\circ} \mathrm{C}$. These antibodies were as follows: hnRNPK, LC-3B, HDAC6, p62, $\alpha$-TAT1, $\alpha$-tubulin, $\beta$-tubulin, acetylated $\alpha$-tubulin and GAPDH. Next, membranes were washed with TBST buffer and incubated with horseradish peroxidase-conjugated secondary antibodies (dilution, 1:5,000; cat. no. SA00001-1 or SA00001-2; ProteinTech Group, Inc.) for $60 \mathrm{~min}$ at room temperature. All antibodies used in the present study were diluted in 5\% dry skimmed milk in TBST buffer. An enhanced chemiluminescence kit (Beyotime Institute of Biotechnology) was used to detect the expression of proteins. GAPDH, $\alpha$-tubulin or $\beta$-tubulin quantification was used to correct for variations in total protein loading. The western blotting bands were quantified using ImageJ software (version number, 1.42; National Institutes of Health, Bethesda, MD, USA).

Reverse transcription-quantitative polymerase chain reaction $(R T-q P C R)$. Total RNA was extracted from cultured cells using TRIzol reagent (Thermo Fisher Scientific, Inc.) and DNA was removed using the recombinant DNaseI. cDNA was prepared from $1 \mu \mathrm{g}$ total RNA, using reverse transcriptase and an iScript ${ }^{\mathrm{TM}}$ cDNA synthesis kit (cat. no. 1708891; Bio-Rad Laboratories, Inc., Hercules, CA, USA) according to the manufacturer's protocol. All PCR reactions were performed using SsoFast $^{\mathrm{TM}}$ Eva Green Supermix (cat. no. 172-5202; Bio-Rad
Laboratories, Inc.), with the following 39 thermal cycles: $95^{\circ} \mathrm{C}$ for $15 \mathrm{sec} ; 60^{\circ} \mathrm{C}$ for $15 \mathrm{sec}$; and $72^{\circ} \mathrm{C}$ for $20 \mathrm{sec}$. The following primers were used for PCR: HDAC6 forward, 5'-GGCTTCAG TTTCCTGTGCTC-3' and reverse, 5'-CTTCCTCCTCGCTC TCCTCT-3'; and GAPDH (control) forward, 5'-AACGGATTT GGTCGTATTG-3' and reverse, 5'-GGAAGATGGTGATGGG ATT-3'. The relative concentration of HDAC6 mRNA was calculated using the $2^{-\Delta \Delta \mathrm{Cq}}$ method (37-39).

Confocal immunofluorescence. Cells cultured on glass coverslips were washed with PBS, fixed with $4 \%$ paraformaldehyde in PBS for $30 \mathrm{~min}$ at room temperature, washed with $2 \mathrm{mg} / \mathrm{ml}$ glycine in PBS, permeabilized with $0.2 \%$ Triton X-100 for 10 min and blocked with $10 \%$ goat serum in PBS for $60 \mathrm{~min}$ at room temperature. The cells were subsequently incubated with primary antibodies diluted at 1:100 in 2\% goat serum PBS overnight at $4^{\circ} \mathrm{C}$. Following extensive washes with a washing buffer (0.05\% Tween-20, $1 \%$ BSA, PBS), cells were incubated with fluorescent secondary antibodies (dilution, 1:200) for an additional $60 \mathrm{~min}$ in the dark at room temperature and washed again with a washing buffer. These fluorescent secondary antibodies were as follows: Alexa Fluor 594 goat anti-rabbit IgG (cat. no. ZF-0516; ZSGB-BIO Co., Ltd., Beijing, China), Alexa Fluor 594 goat anti-mouse IgG (cat. no. ZF-0513; ZSGB-BIO Co., Ltd.), Alexa Flour 488 goat anti-rabbit IgG (cat. no. ZF-0511; ZSGB-BIO Co., Ltd.) and Alexa Fluor 488 goat anti-mouse IgG (cat. no. ZF-0512; ZSGB-BIO Co., Ltd.). The nuclei were counterstained with 4, 6-diamidino-2-phenylindole (Sigma-Aldrich; Merck KGaA) for $10 \mathrm{~min}$ at room temperature and washed with PBS. The imaging experiments were performed using laser scanning confocal microscopes (LSM700; Zeiss GmbH, Jena, Germany) equipped with a Zeiss Plan-Neofluar 40x NA 0.75 or 63x NA 1.25 Oil Dic objective. The magnification was $\mathrm{x} 400$ or $\mathrm{x} 630$.

Statistical analysis. All statistical analyses were performed using GraphPad Prism 6 software (GraphPad Software, Inc., La Jolla, CA, USA). The significant differences between two groups were analyzed by the two-tailed unpaired Student's t-tests $(40,41)$. Significant differences between multiple groups were analyzed by two-way analysis of variance and Holm-Sidak multiple comparisons tests. Data are expressed as the mean \pm standard deviation, $n \geq 3$, unless otherwise stated. $\mathrm{P}<0.05$ was considered to indicate a statistically significant difference.

\section{Results}

hnRNPK negatively regulates basal autophagy in 293 cells. To investigate the role of hnRNPK in autophagy, hnRNPK was either knocked down with a specific siRNA or overexpressed by transfection with a Flag-hnRNPK plasmid in 293 cells. A total of $48 \mathrm{~h}$ after transfection, autophagy flux activation was assessed by the ratio of LC3B-II/LCB3-I using western blotting with a specific anti-LC3B antibody. As shown in Fig. 1A and B, hnRNPK-knockdown significantly increased the LC3B-I to LCB3-II conversion, whereas its overexpression inhibited this process, suggesting a negative role of hnRNPK in the basal autophagy of 293 cells. Furthermore, it was found that LC3-II, the lipidated form of LC3 and an autophagosome marker, was increased by hnRNPK-knockdown and -overexpression. 

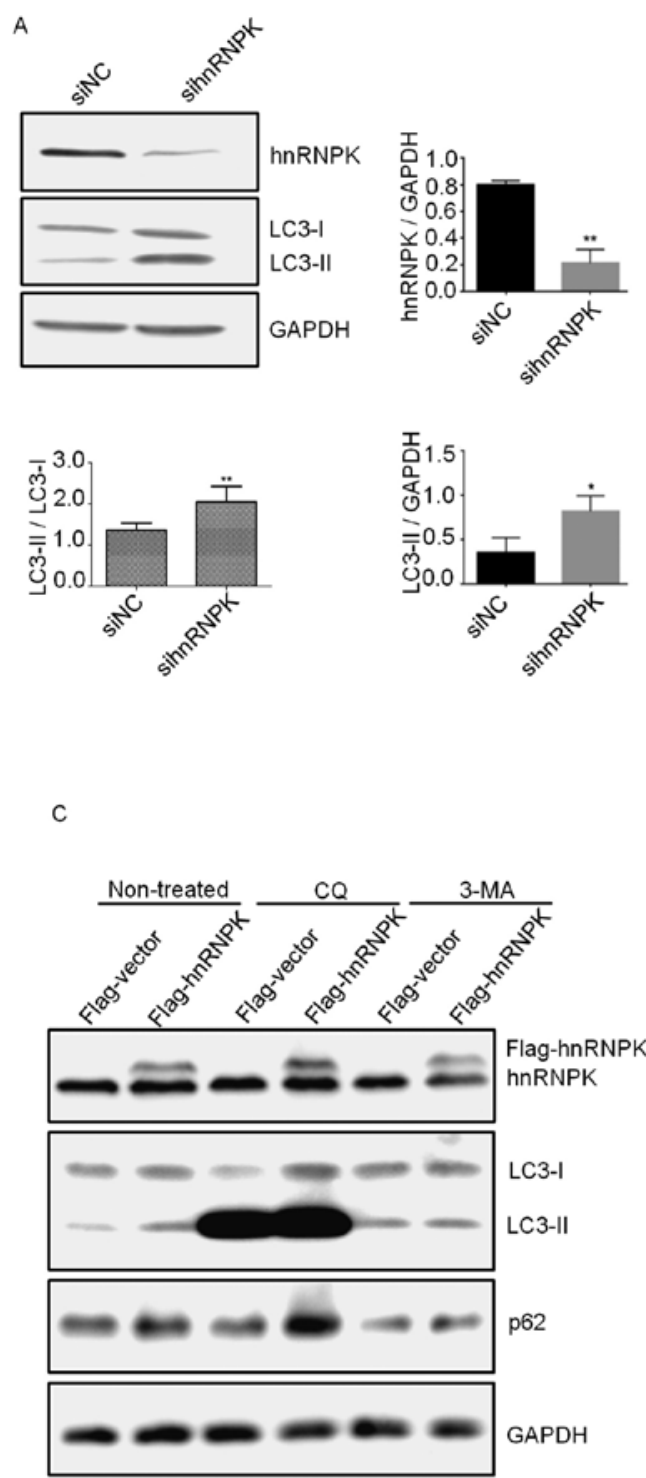

B
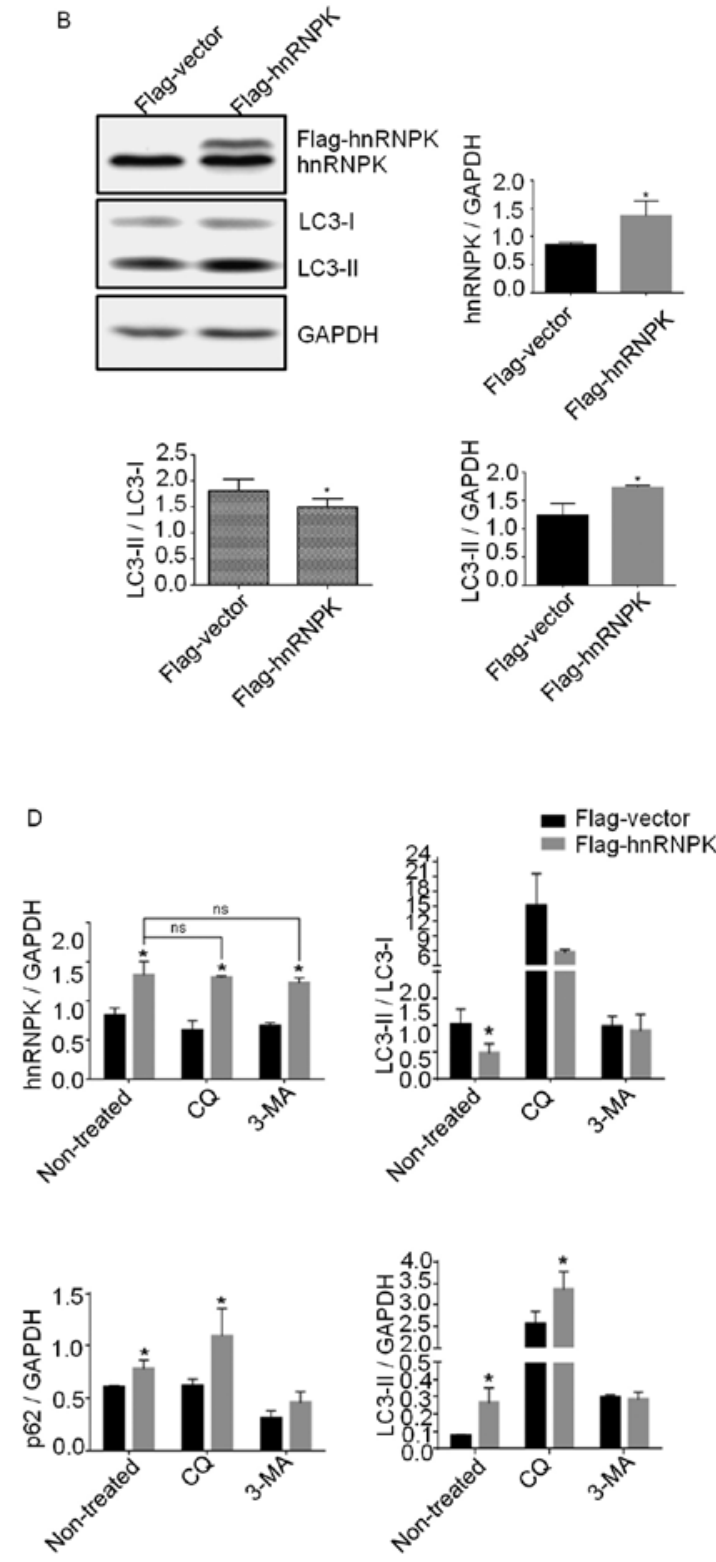

Figure 1. hnRNPK negatively regulates basal autophagy. 293 cells were transfected with (A) sihnRNPK or (B) Flag-hnRNPK and their respective negative controls, and analyzed by western blotting. The signal intensity from the LC3B immunoblot was quantified. The ratios of LC3-II/LC3-I and LC3-II/GAPDH were calculated and presented as the average values of the means from three independent experiments along with the standard deviation. ${ }^{*} \mathrm{P}<0.05$ and ${ }^{* *} \mathrm{P}<0.01$. (C) 293 cells were transfected with pCMV-flag-hnRNPK or pCMV-flag-vector for $24 \mathrm{~h}$, treated with 3-MA or CQ, and harvested for western blot analysis using the indicated antibodies. (D) Quantification of the signal intensity from the LC3B and p62 immunoblots of 293 cells treated as described in (C). GAPDH was used as an internal control for the normalization of the values. The ratios of LC3-II/LC3-I and p62/GAPDH reflect the degree of the autophagic flux, and LC3-II/GAPDH indicates autophagic membranes. The results are presented as the mean \pm standard deviation of three independent experiments.

Taken together, the aforementioned experimental results suggested that hnRNPK may inhibit autophagy flux in the late stage of the process at the level of lysosomal degradation. In order to further confirm this result and determine the precise function of hnRNPK in the autophagic flux, experiments were performed by combining hnRNPK-overexpression with two autophagy inhibitors, including 3-MA, which inhibits the formation of autophagic vacuoles at the initial stage of the autophagic process, and CQ, an inhibitor of autophagy at the late stage, which blocks autophagosome fusion and degradation. 293 cells were transfected with pCMV-flag-hnRNPK or pCMV-flag empty vector for $24 \mathrm{~h}$ and then treated with $25 \mu \mathrm{M}$ CQ or $1 \mathrm{mM} 3-\mathrm{MA}$. The results indicated that hnRNPKoverexpression could reverse the inhibitory effect of CQ on autophagy, whereas 3-MA-inhibited autophagy remained unaltered (Fig. 1C and D). A double band could be seen on the western blot when cells were transfected with pCMV-flaghnRNPK and incubated with the hnRNPK antibody. The protein band of lower molecular weight represented the endogenous hnRNPK, while the upper band represented the exogenous flag-hnRNPK, with the quantification of hnRNPKoverexpression shown in Fig. 1B. In addition, the increased levels of p62 further confirmed the inhibition of selective autophagy via hnRNPK-overexpression. This result suggested that hnRNPK may serve a role in autophagy at the stage involving autophagosome fusion.

Subsequently, the role of hnRNPK in serum starvationor rapamycin- induced autophagy was investigated. Serum 
A

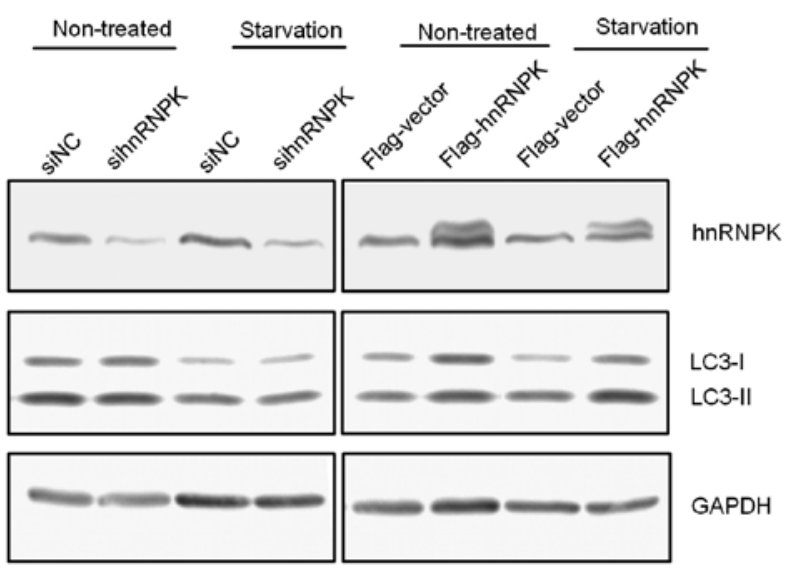

c

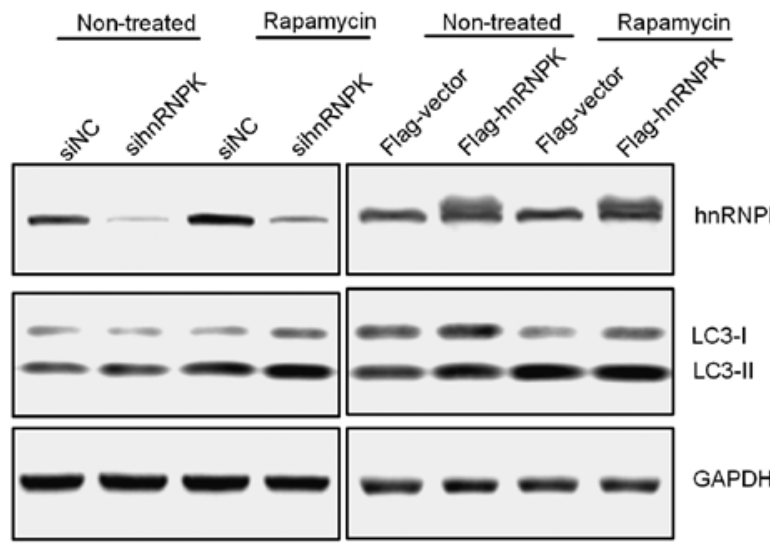

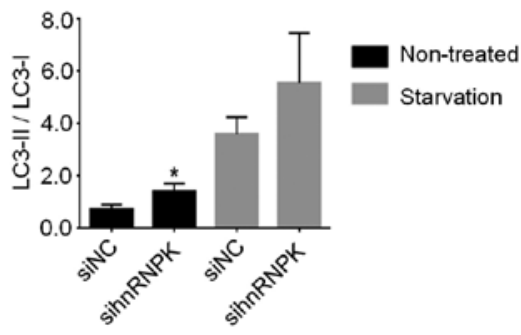
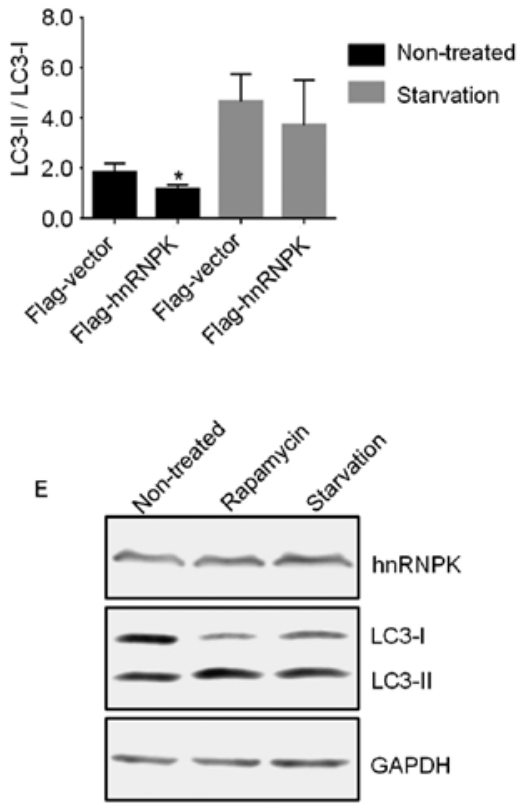
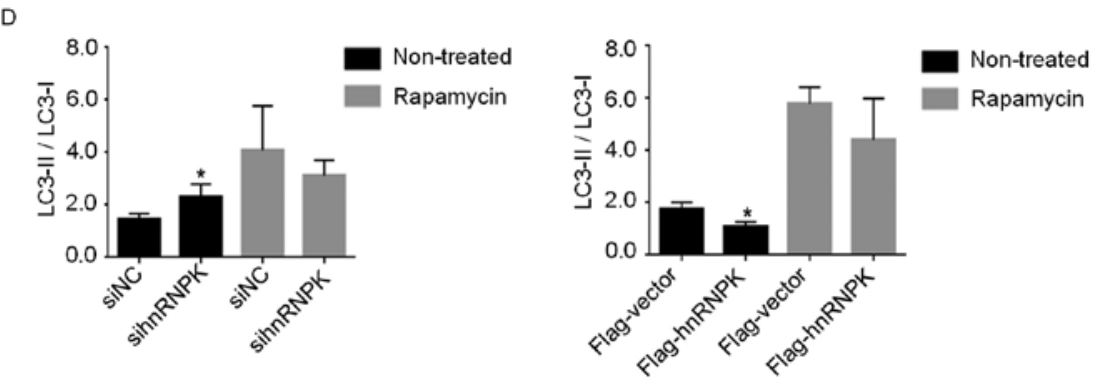

Figure 2. Neither knockdown nor overexpression of hnRNPK exert marked effects on serum starvation- or rapamycin-induced autophagy. (A) 293 cells were transfected with either specific hnRNPK-targeting siRNA or pCMV-flag-hnRNPK plasmid, prior to being subjected to serum starvation for $6 \mathrm{~h}$, and the proteins were subsequently extracted and analyzed by western blotting. (B) The signal intensity of LC3-I and LC3-II protein bands in (A) were quantified, and the ratio of LC3-II/LC3-I was calculated. The results are presented as the mean \pm standard deviation of three independent experiments. (C) 293 cells were transfected with either specific hnRNPK-targeting siRNA or respective negative control, prior to being treated with rapamycin for $24 \mathrm{~h}$, and the proteins were extracted and analyzed by western blotting. (D) The signal intensity of LC3-I and LC3-II protein bands in (C) were quantified, and the ratio of LC3-II/LC3-I was calculated. The results are presented as the mean \pm standard deviation of three independent experiments. (E) 293 cells subjected to rapamycin treatment or serum starvation were analyzed by western blotting. The conversion of LC3B-I to LC3B-II was significantly increased in cells treated with rapamycin and serum starvation, while the endogenous expression of hnRNPK remained unchanged.

starvation and rapamycin are the two most commonly used conditions for the induction of non-selective autophagy in which cytosolic contents and organelles are degraded to supply cells with essential macromolecules, and to provide the energy required for survival. 293 cells were either transfected with specific hnRNPK-targeting siRNA or pCMV-flag-hnRNPK plasmid for $24 \mathrm{~h}$, and then treated with $100 \mathrm{nM}$ rapamycin for $24 \mathrm{~h}$ or subjected to serum starvation for $6 \mathrm{~h}$. Cells were subsequently collected and subjected to western blotting for the verification of hnRNPK-knockdown or -overexpression and determination of the expression levels of LC3-I and LC3-II.
Notably, compared with their respective negative controls, as indicated, neither knockdown nor overexpression of hnRNPK in 293 cells exerted notable effects on the serum starvation- or rapamycin-induced autophagy, as demonstrated in Fig. 2A-D. In addition, the present study verified that while rapamycin and serum starvation induced significant autophagy in the treated 293 cells, these treatments had no notable effect on the endogenous expression of hnRNPK (Fig. 2E), further excluding the possible involvement of hnRNPK in these two non-selective autophagy processes. Taken together, these results suggested that hnRNPK is likely to be involved in nutrient-independent 


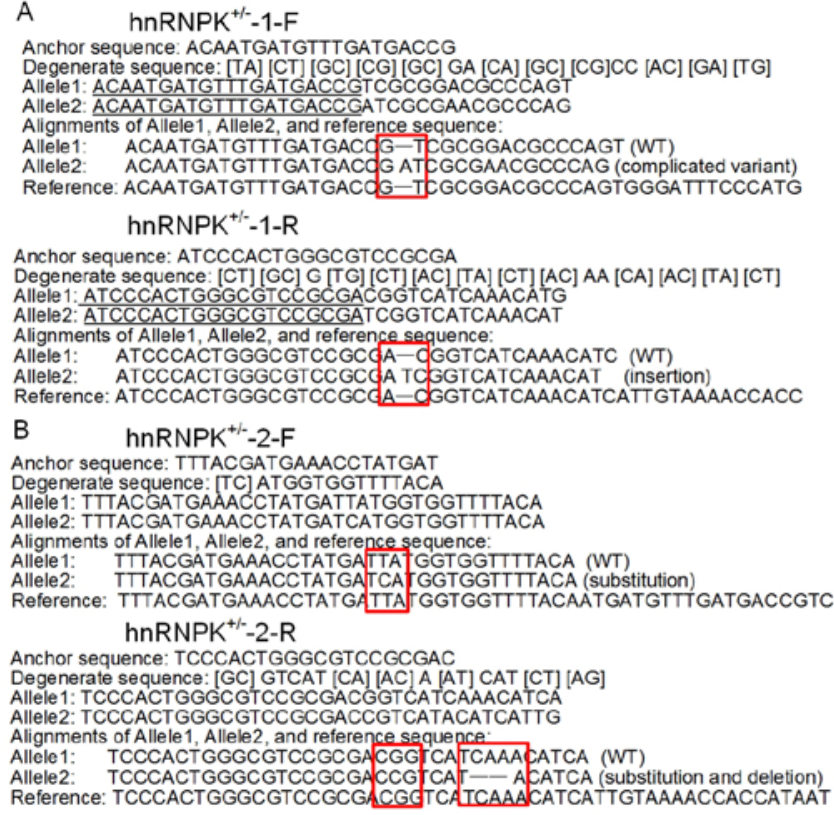

C

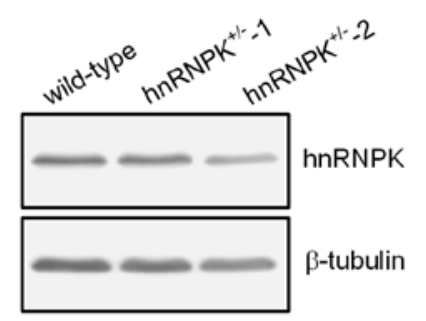

$\mathrm{E}$

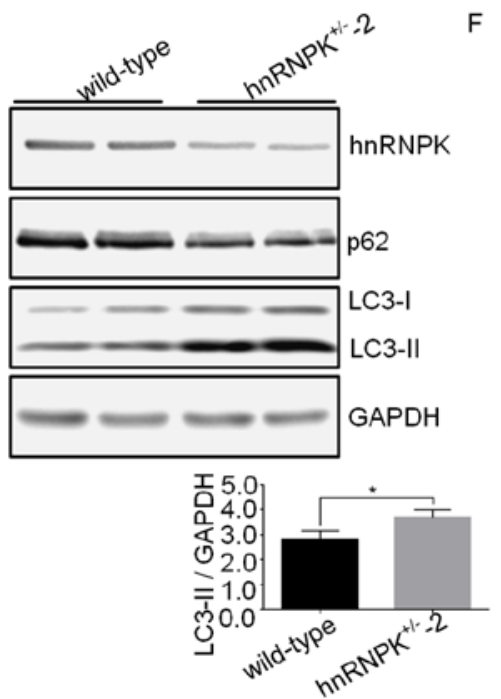

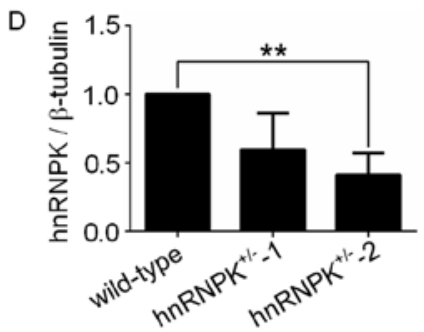
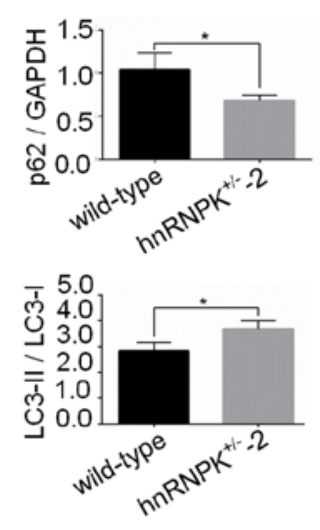
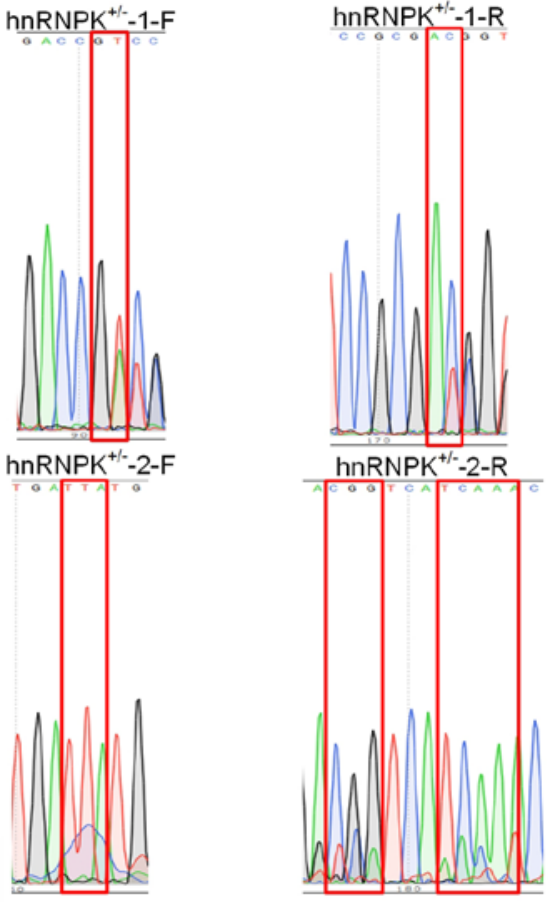

G
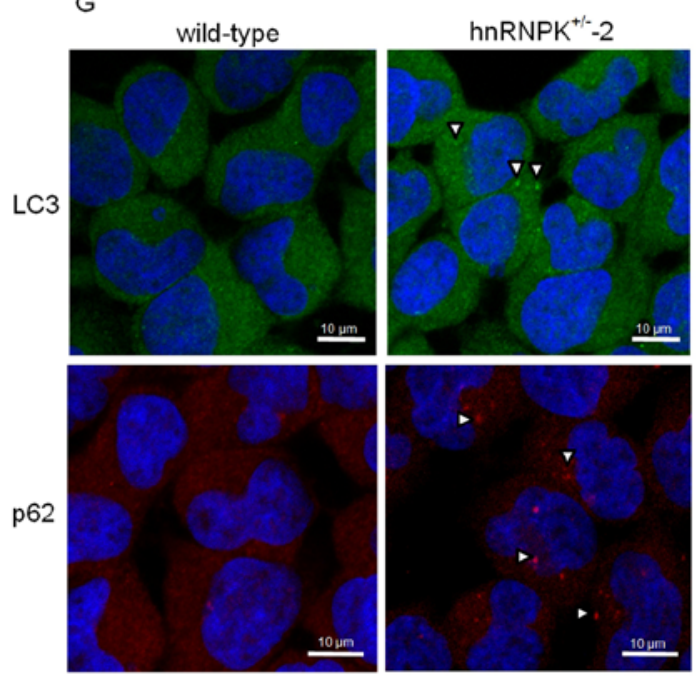

Figure 3. The hnRNPK ${ }^{+-}$cells exhibit increased levels of basal autophagy. Genomic sequences of the hnRNPK ${ }^{+/}$cells generated using the CRISPR-Cas9 system. (A) Allele- 1 of the hnRNPK ${ }^{+/}-1$ clone displayed a 2 base-pair insertion and (B) allele- 2 of the hnRNPK ${ }^{+/}-2$ clone contained a deletion of 3 base-pairs and a 2 base-pair replacement, indicated by the red frames. (C) Western blot analysis showing the effect of the hnRNPK-knockdown in hnRNPK ${ }^{+-}$cells. The results shown are representative of at least three independent experiments. (D) The signal intensity from the hnRNPK immunoblot was quantified. The results are presented as the fold-changes following normalization using $\beta$-tubulin and compared with the control group. Data are presented as the mean \pm standard deviation, $\mathrm{n} \geq 3,{ }^{* *} \mathrm{P}<0.01$. The error bar represents the standard deviation. (E) Western blotting showing the autophagy status in the hnRNPK ${ }^{+/}-2$ cells. (F) Quantification of signal intensities from the LC3B and p62 immunoblots and the ratios of LC3-II/LC3-I, LC3-II/GAPDH and p62/GAPDH are presented as the mean \pm standard deviation of three independent experiments. (G) The hnRNPK ${ }^{+/}-2$ and the parental 293 cells were immunostained with anti-LC3B (green), anti-p62 antibody (red) and DAPI (blue). Arrowheads indicated LC3-positive dots or p62 punctae. Scale bars=10 $\mu \mathrm{m}$.

basal autophagy by intervening at the later stage, but is dispensable in serum starvation- or rapamycin-induced autophagy.

Establishment of a stable hnRNPK KO 293 cell line. In order to further confirm the function of hnRNPK in basal autophagy and provide a stable cell model for the associated mechanistic studies, a hnRNPK KO 293 cell line was generated using the prokaryotic type II CRISPR-Cas 9 system $(42,43)$. Two clones have been isolated through selection with puromycin. The first clone (hnRNPK ${ }^{+/}-1$ ) displayed a 2 base-pair insertion (Fig. 3A), 
whereas the other clone (hnRNPK ${ }^{+/-}$-2) contained a deletion of 3 base pairs and a 2 base-pair replacement (Fig. 3B). The two clones are single allele $\mathrm{KO}$ clones. The fact that only single allele $\mathrm{KO}$ clones could be recovered may be explained by the essential role of hnRNPK in the survival of the cell, in which case the complete depletion of hnRNPK may have been lethal. In support of this hypothesis, it has been recently reported that double-allele KO of hnRNPK was lethal for the mouse embryo (27). Due to the single allele KO in the two clones used in the present study, a decrease in the hnRNPK expression levels rather than complete depletion was expected. This was verified by the western blotting shown in Fig. 3C and D, where an improved knockdown efficiency of $\mathrm{hnRNPK}^{+/-}-2$ has been observed compared with the hnRNPK ${ }^{+/}-1$ clone. Sequencing analysis revealed that, in allele 2 of the $\mathrm{hnRNPK}^{+/-}-2$ cells, 2 amino acid changes occurred at Y236 and D245 positions, along with the deletion of the F243 amino acid residue. Y236 is a binding site between hnRNPK and tyrosine kinases, including Src, Lyn, Fyn and Lck, mostly involved in gene expression and signal transduction (17). F243 and D245 were located in the $\mathrm{K}$-protein-interactive region $(\mathrm{KI})$ responsible for a number of the known $\mathrm{K}$ protein interactions $(11,24)$. These mutations did not result in a frameshift; however, they may lead to the conformational alteration and instability of the hnRNPK protein. hnRNPK ${ }^{+-}-2$ was therefore selected for the subsequent experiments. The autophagy status in the hnRNPK ${ }^{+-}-2$ cells was firstly examined by western blotting. As shown in Fig. 3E and F, significant augmentation of the LC3-II /LC3-I ratio and LC3-II protein level, along with a decrease in the p62 level could be detected in the hnRNPK ${ }^{+/-} 2$ cells, compared with the wild-type cells. Furthermore, more prominent LC3-positive dots and p62 punctae were observed in hnRNPK ${ }^{+/}-2$ cells by immunofluorescence (Fig. 3G). These results further confirmed the important role of hnRNPK in the basal autophagy of 293 cells.

hnRNPK inhibits the expression of HDAC6 to maintain $\alpha$-tubulin K40 acetylation in basal autophagy. The present study subsequently aimed to investigate the mechanism of action of hnRNPK in autophagy under normal nutrient conditions. Our previous study had demonstrated that hnRNPK regulated the $\alpha$-tubulin K40 acetylation in multi-nucleated mature osteoclasts (30). As expected, it was revealed that the $\alpha$-tubulin K40 acetylation level was significantly decreased in the hnRNPK ${ }^{+/}-2$ cells compared with the wild-type 293 cells, while the total $\alpha$-tubulin protein level remained unchanged (Fig. 4A and B). $\alpha$-tubulin K40 acetylation is mainly regulated by two enzymes with opposite functions, the acetyltransferase $\alpha$-TAT and the deacetylase HDAC6 $(44,45)$. Based on the function of hnRNPK in the regulation of gene expression, the present study further examined if hnRNPK may influence $\alpha$-tubulin K40 acetylation through regulating the expression of these two enzymes. Western blot assays were performed with protein extracts from the wild-type 293 and hnRNPK ${ }^{+-}-2$ cells using specific anti- $\alpha$-TAT or anti-HDAC6 antibodies. It was revealed that, while hnRNPK deficiency had no effect on the $\alpha$-TAT protein expression level, the HDAC6 protein level was notably increased in the hnRNPK ${ }^{+-}-2$ cells, compared with the wild-type 293 cells in basal autophagy. This result was subsequently confirmed by RT-qPCR, which indicated a 2.5 -fold increase in the HDAC6 mRNA level in the hnRNPK ${ }^{+/}-2$ cells compared with the wild-type 293 cells, suggesting that hnRNPK regulated the activity of HDAC6 by modulating its mRNA synthesis or metabolism, thereby controlling the acetylation of $\alpha$-tubulin K40 (Fig. 4C). In order to determine whether autophagy regulation by the hnRNPKHDAC6 axis is an event specific to basal autophagy, the wild-type 293 cells and hnRNPK ${ }^{+/}-2$ cells were treated with rapamycin or subjected to serum starvation, and subsequently collected and subjected to qPCR and western blot analyses for the assessment of the HDAC6 expression levels. As shown in Fig. 4D-I, neither mRNA nor protein levels of HDAC6 in the wild-type or hnRNPK ${ }^{+/}-2293$ cells exhibited marked changes in the serum starvation- or rapamycin-induced autophagy. In accordance with these results, the hnRNPK single allele KO had no significant effect on the $\alpha$-tubulin K40 acetylation and $\alpha$-TAT expression level in the serum starvation- or rapamycininduced autophagy. All these results further supported the hypothesis that the hnRNPK-HDAC6 axis contributes toward basal autophagy but not toward serum-starvation or rapamycin-induced autophagy.

Immunofluorescence detection was used to confirm the decrease in $\alpha$-tubulin acetylation in cells. Furthermore, it was demonstrated that this alteration stemmed from the hyperactivity of HDAC6 since the treatment of cells with Tub A, a selective inhibitor of HDAC6, could re-stimulate the $\alpha$-tubulin K40 acetylation level (Fig. 5A). As expected, variations in the $\alpha$-tubulin acetylation of the parental 293 cells, hnRNPK ${ }^{+/-}-2$ cells and Tub A treated hnRNPK ${ }^{+/-}-2$ cells was associated with the degree of autophagy of the cells, as indicated by the LC3-II/LC3-I ratio, and the expression levels of LC3-II and p62 determined by western blotting (Fig. 5B and C).

hnRNPK-HDAC6 axis regulates autophagosome-lysosome fusion associated with QC autophagy. Autophagosomes must fuse with lysosomes to degrade the aggregates, and HDAC6 is required for an efficient fusion of autophagosomes and lysosomes in mouse embryonic fibroblasts (MEFs) (3). In order to determine whether hnRNPK may serve a role in such a process, the mCherry-GFP-LC3 reporter assay was used to evaluate the autophagosome-lysosome fusion efficiency in the wild-type 293 cells and hnRNPK ${ }^{+-}-2$ cells treated or untreated with Tub A. As shown in Fig. 6A, the number of yellow fluorescence-labelled vesicles was decreased, while an increased number of red fluorescence-labeled vesicles appeared in the hnRNPK ${ }^{+/}-2293$ cells compared with the wild-type control cells. This result indicated an increased efficiency of lysosome-autophagosome fusion (Fig. 6A, upper and middle panels). Accordingly, autophagosomelysosome fusion was effectively suppressed when the hnRNPK ${ }^{+-}-2$ cells were treated with Tub A, as indicated by the increased appearance of yellow fluorescence-labelled vesicles compared with cells untreated with Tub A (Fig. 6A, middle and lower panels). Accumulation of red vesicles in the hnRNPK ${ }^{+-}-2$ cells strongly indicated that HDAC6 is required for efficient autophagosome-lysosome fusion under normal nutrient conditions (Fig. 6B), which was in agreement with a previous study (3). These results demonstrated that the effect of hnRNPK on autophagosome-lysosome fusion is HDAC6dependent. 
A

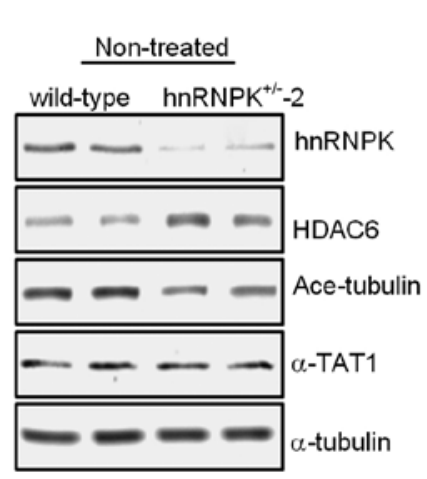

D

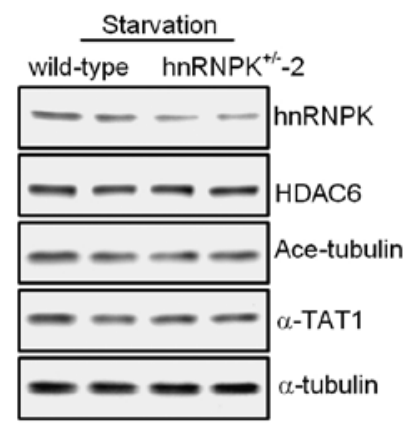

G

Rapamycin

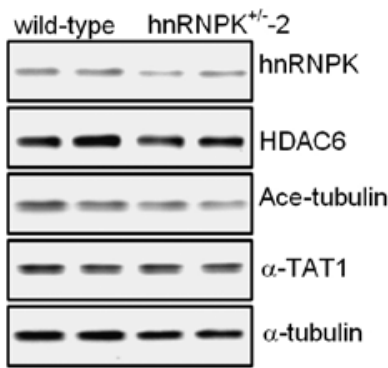

B

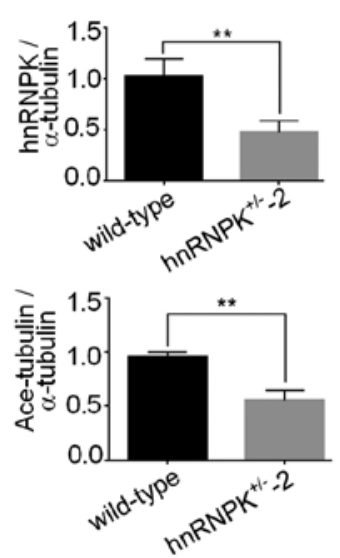

E

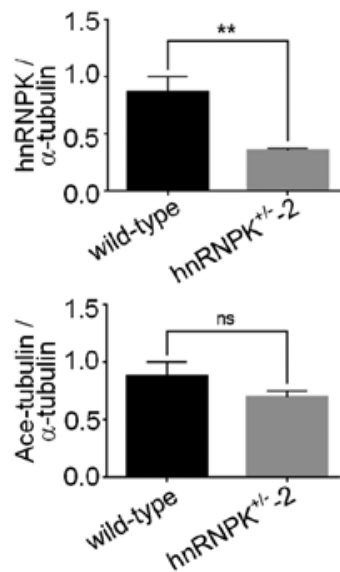

H

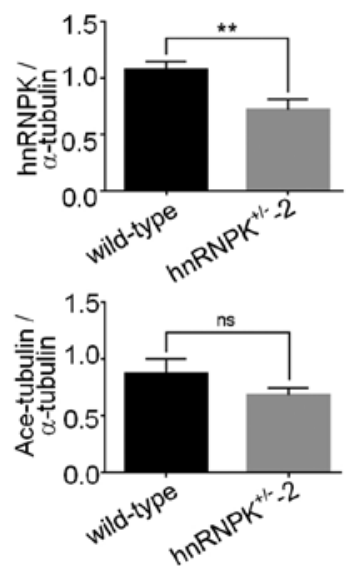

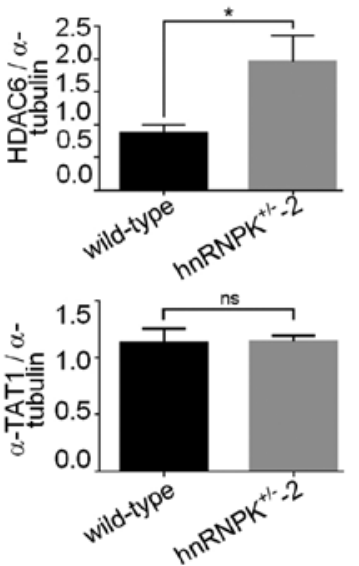

C

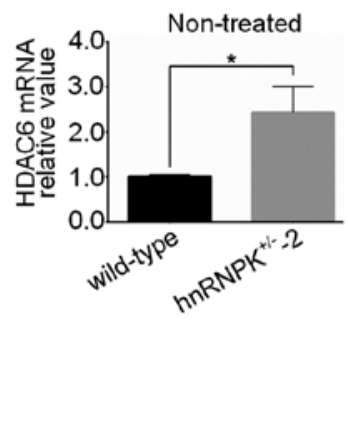

F
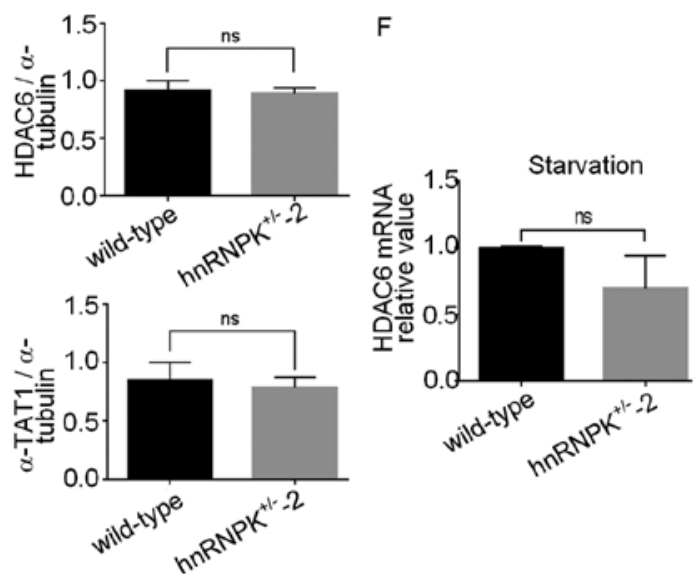

I
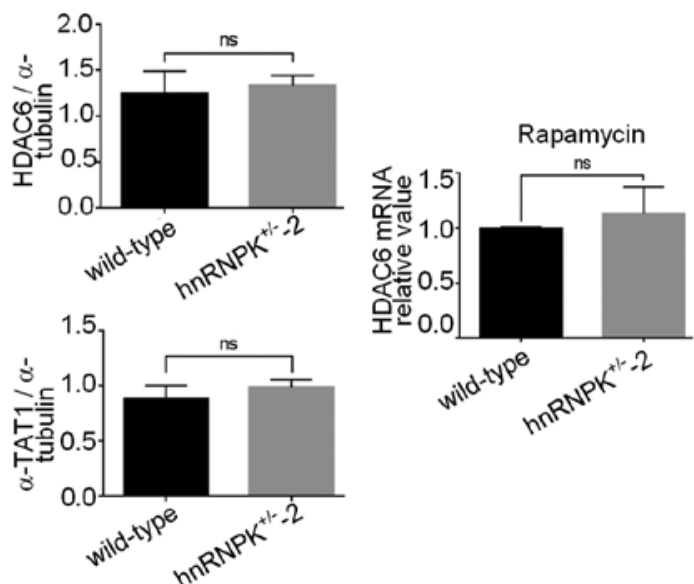

Figure 4. hnRNPK inhibits HDAC6 expression to maintain $\alpha$-tubulin K40 acetylation in basal autophagy. (A) Western blot analyses were performed with proteins extracted from the wild-type 293 and hnRNPK ${ }^{+/}-2$ cells under normal nutrient conditions using the indicated antibodies. (B) The signal intensity from the immunoblot as described in (A) was quantified and $\alpha$-tubulin was used for the normalization of the expression value. Data are presented as the mean \pm standard deviation, $\mathrm{n} \geq 3$. ${ }^{*} \mathrm{P}<0.05,{ }^{* *} \mathrm{P}<0.01$; ns, no significance. The error bars represent the standard deviation. (C) Total mRNA was extracted from the wild-type or hnRNPK ${ }^{+/}-2$ cells, and HDAC6 mRNA levels were quantified by reverse transcription-quantitative polymerase chain reaction. The results are presented as the fold-changes following normalization using GAPDH and compared with the control group. Data are presented as the mean \pm standard deviation, $\mathrm{n} \geq 3,{ }^{*} \mathrm{P}<0.05$. The error bars represent the standard deviation. 293 cells and hnRNPK ${ }^{+/}-2$ cells were (D) subjected to serum starvation for $6 \mathrm{~h}$ or (G) treated with rapamycin for $24 \mathrm{~h}$, and protein expression levels were analyzed by western blotting using the indicated antibodies. The signal intensities from the immunoblots as described in (D) and (G) were quantified, and $\alpha$-tubulin was used for the normalization of the value. The results are presented as the mean \pm standard deviation of three independent experiments $(\mathrm{E}$ and $\mathrm{H})$. $(\mathrm{H})$ Total mRNA was extracted from the wild-type or hnRNPK ${ }^{+/}-2$ cells described in (D and G), and HDAC6 mRNA levels were quantified by reverse transcription-quantitative polymerase chain reaction. The results are presented as the fold-changes following normalization using GAPDH and compared with the control group ( $\mathrm{F}$ and I). Data are presented as the mean \pm standard deviation, $\mathrm{n} \geq 3$, ${ }^{*} \mathrm{P}<0.05$. The error bars represent the standard deviation.

It has been hypothesized that QC autophagy removes toxic protein aggregates and that cargo selection is partly achieved by targeted ubiquitination for degradation (46-48). To further demonstrate the functional role of hnRNPK in protein 
A
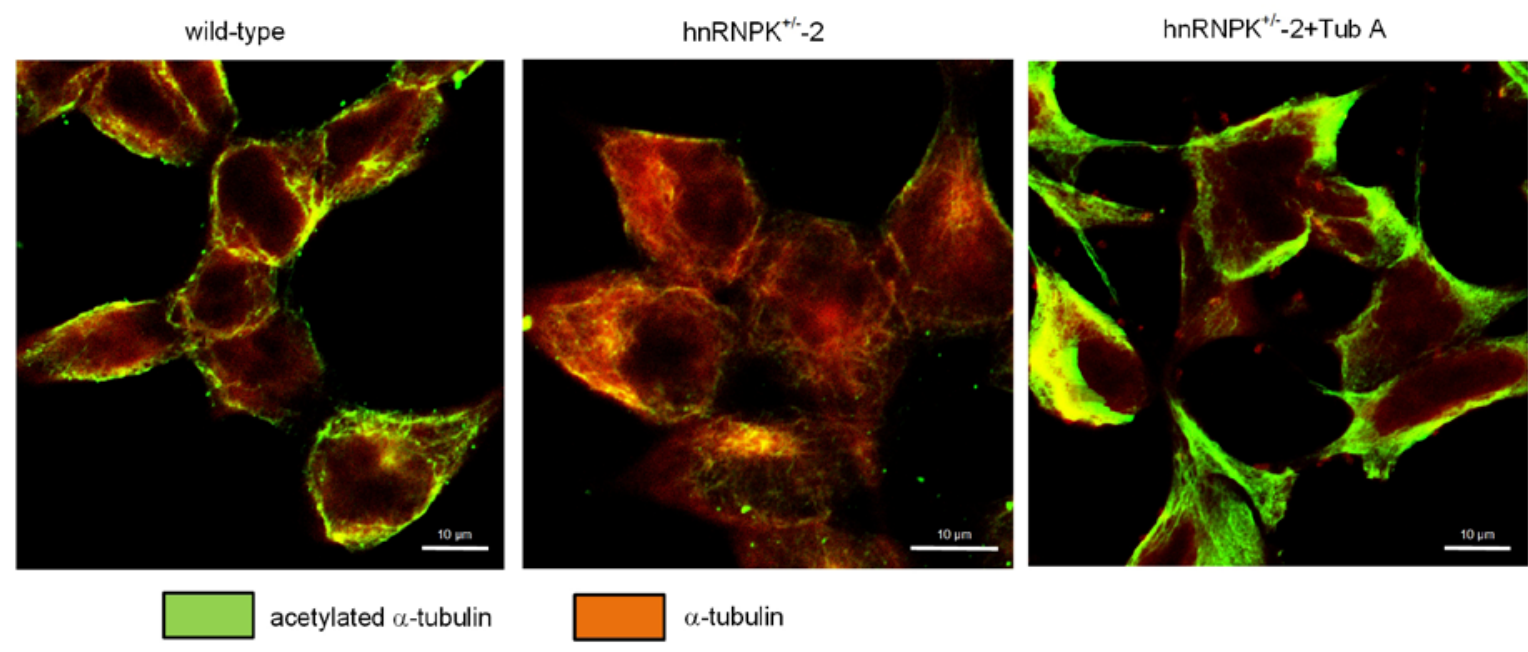

B

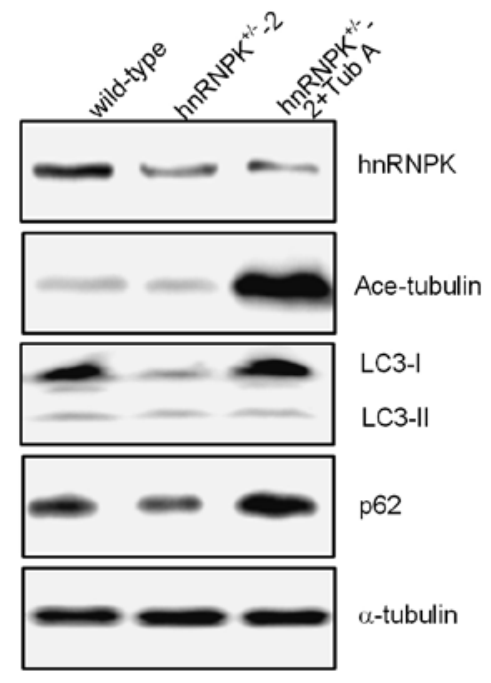

C
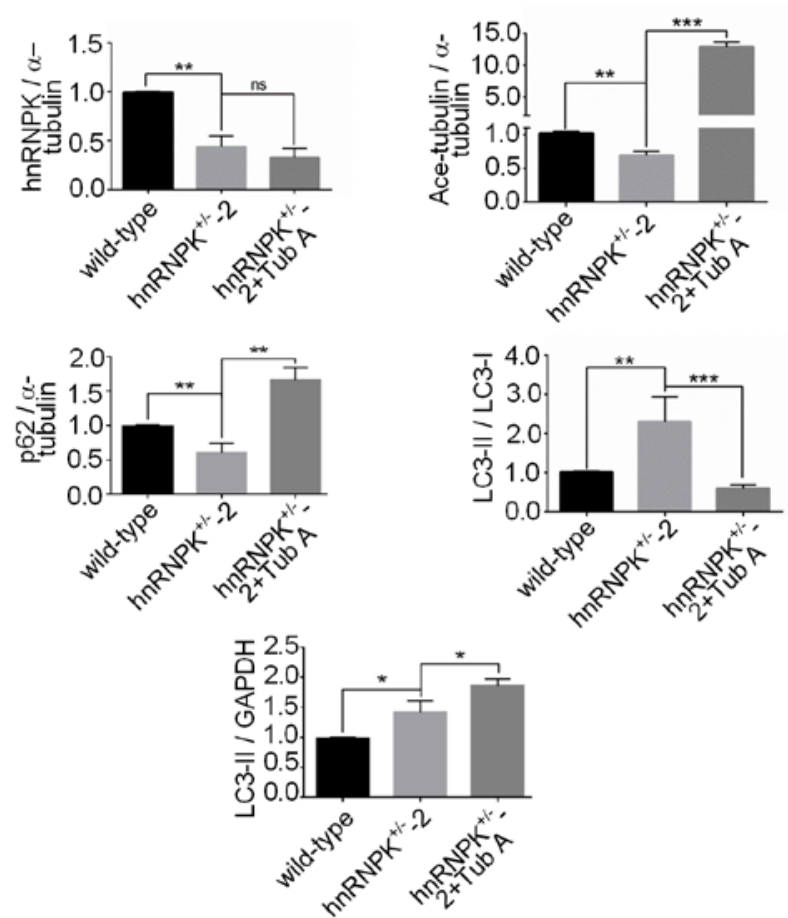

Figure 5. hnRNPK modulates autophagy control through the downregulation of the expression levels of HDAC6. (A) Wild-type 293 cells, hnRNPK ${ }^{+/}-2$ cells or hnRNPK ${ }^{+/}-2$ cells treated with Tub A were stained with antibodies against K40 acetylated $\alpha$-tubulin (green) or Tubulin-Tracker (red). Scale bars=10 $\mu \mathrm{m}$. (B) Western blotting showing the degree of autophagy of the cells used in (A), represented by the protein levels of LC3-II and p62, and the LC3-II/LC3-I ratio. (C) Signal intensities from the immunoblot described in (A) were quantified. The results are presented as the fold-changes following normalization using $\alpha$-tubulin and compared with the control group. Data are presented as the mean \pm standard deviation. ${ }^{* * *} \mathrm{P}<0.001,{ }^{* *} \mathrm{P}<0.01,{ }^{*} \mathrm{P}<0.05$; ns, no significance. The error bars represent the standard deviation.

aggregate clearance of QC autophagy, an immunofluorescence assay was used to label the LC3-positive autophagosomes and the ubiquitinated positive protein aggregates in the 3 aforementioned types of cells under the treatment of MG132 to block the proteasome degradation. As shown in Fig. 6C, compared with the control cells, prominent LC3-positive autophagic vesicles (green dots) were abundantly present and found co-localized with the ubiquitin-positive protein aggregates (red dots) in the hnRNPK ${ }^{+/-}-2293$ cells, indicating a highly efficient selective autophagy-dependent protein aggregate degradation (Fig. 6C, middle panels). When the same cells were treated with Tub A, a comparable intensity of the LC3-positive autophagosome staining could be observed; however, this staining was not co-localized with the ubiquitin-positive aggregates, likely due to the deficiency in docking and tethering ubiquitinated protein aggregates into autophagosomes. Taken together, the results of the present study suggested that hnRNPK could regulate the aggregate clearance in the QC autophagy through a HDAC6-dependent mechanism. 
A
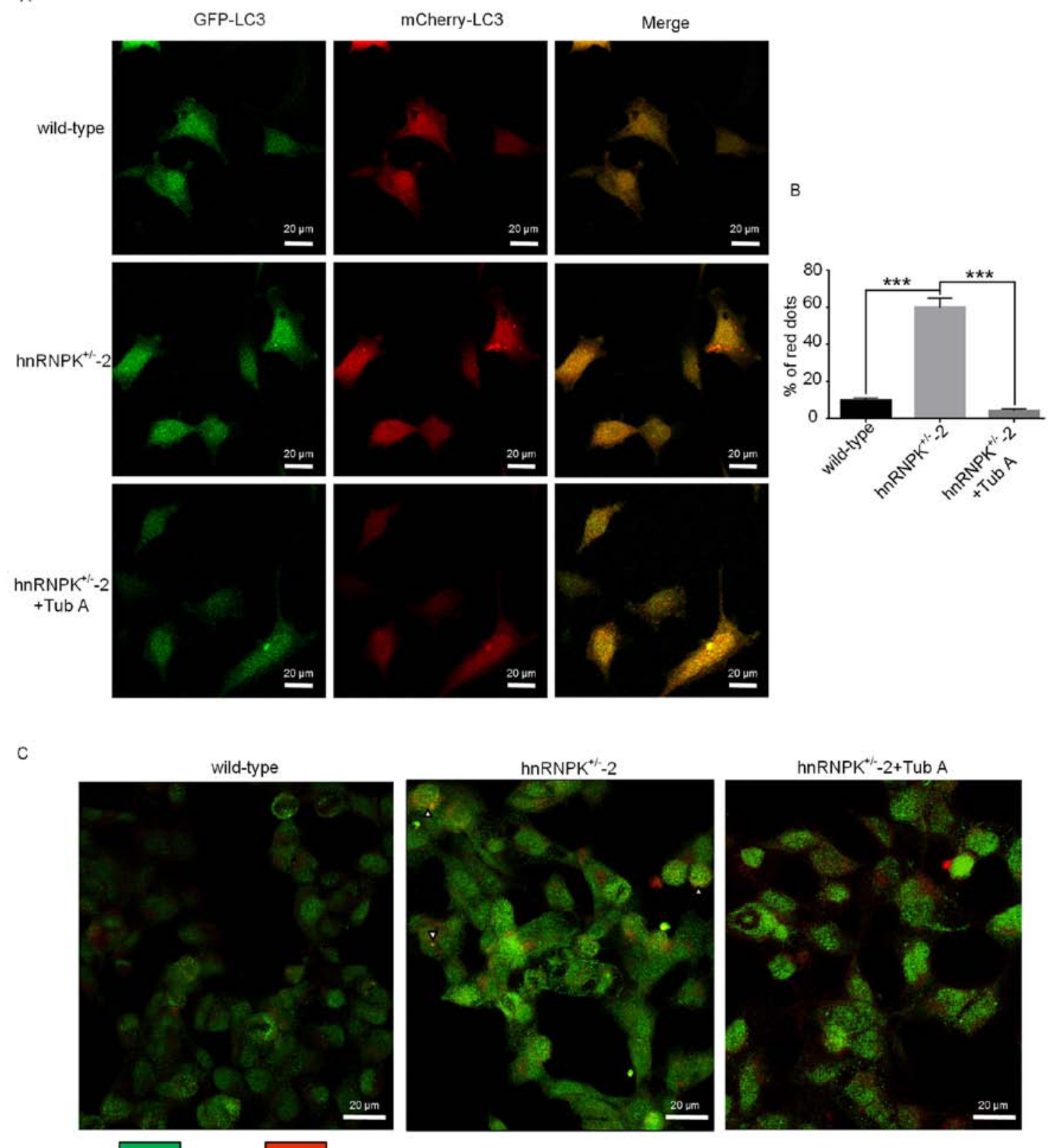

$\square$ LC3

ubiquitin

Figure 6. hnRNPK regulates autophagosome-lysosome fusion associated with QC autophagy. (A) Wild-type 293, hnRNPK ${ }^{+/}-2$ and hnRNPK ${ }^{+/}-2$ cells treated with Tub A were transfected with a plasmid expressing mCherry-GFP-LC3B fusion protein. Yellow signals indicate non-acidic autophagosomes and red signals indicate acidic autophagolysosomes in merged images (the right panel). Scale bars $=20 \mu \mathrm{M}$. (B) The total number of red vesicles was quantified from two independent experiments ( $\geq 8$ cells each) and presented as the percentage of total mCherry-GFP-LC3 dots (red plus yellow) along with the standard deviation. (C) The cells described in (A) were treated with MG132 and immunostained with antibodies against anti-LC3 (green) and ubiquitin (red). White arrows indicate colocalization of ubiquitin-positive aggregates with LC3-positiveautophagosomes. Scale bars $=20 \mu \mathrm{m}$.

\section{Discussion}

Autophagy not only prevents the accumulation of damaged or unnecessary components, but also facilitates the recycling of these components to sustain homoeostasis. The concepts of 'induced autophagy' and 'basal autophagy' have been introduced in several previous studies. While the former produces amino acids in response to extracellular or intracellular stress and signals, including starvation, growth factor deprivation and pathogen infection, the latter of which, also referred to as QC autophagy, is important for baseline turnover of cytosolic components $(9,49)$.
The process of autophagy consists of several sequential steps, including induction, autophagosome formation, degradation and amino acid/peptide generation. The microtubule (MT) cytoskeleton has been reported to be important for all these steps (31,50-53). The MT cytoskeleton consists of highly dynamic tubular polymers assembled from protofilaments of $\alpha / \beta$-tubulin dimers, and is essential for intracellular transport of organelles and macromolecules (33). Previous studies also reported that an intact microtubule cytoskeleton was required for autophagy and that microtubule disruption interfered with autophagy by impairing autolysosome formation $(46,54)$. Kochl et al (31) demonstrated that, in primary rat hepatocytes, 
fusion of autophagosomes with endosomes is inhibited by nocodazole and vinblastine, which prevent the polymerization of microtubules. In particular, protein aggregates are formed in the cytoplasm and accumulate at the microtubule organizing center (MTOC) where they are subsequently engulfed into autophagosomes to subsequently fuse with lysosomes for protein degradation $(46,55)$. K40 acetylated $\alpha$-tubulin is most abundant in stable microtubules, but is absent from dynamic cellular structures; including neuronal growth cones and the leading edges of fibroblasts (36). $\alpha$-tubulin acetylation is mainly regulated by acetyltransferase ( $\alpha$-TAT) and histone deacetylases (HDACs). Previous studies have demonstrated that acetylated microtubules and HDAC6 are required for autophagy (33-36). Consistently, the deacetylase HDAC6 has not only been associated with microtubule-dependent transport of protein aggregates and lysosomes to the MTOC (46), but also with the recruitment and assembly of the cortactin-dependent F-actin cytoskeleton to stimulate the fusion of autophagosomes and lysosomes, suggesting an important role of $\alpha$-tubulin acetylation in these processes. The aforementioned results, together with those from the present study demonstrating that hnRNPK could regulate the dynamic stability of microtubules through upregulating the acetylation of K40 $\alpha$-tubulin in the maturing osteoclasts, support a hypothesis that hnRNPK may serve a critical role in the microtubule-dependent process of autophagy. The results of the present study have not only unraveled the regulatory function of hnRNPK in selective QC autophagy, but also elucidated the associated mechanism by which this protein modulates the expression level of HDAC6 and consequently influences the microtubule dynamics through the regulation of K40 $\alpha$-tubulin acetylation. HDAC6, which deacetylates $\alpha$-tubulin in vivo and in vitro, was often considered a key regulator of the stability of the dynamic microtubules (56). An increasing number of studies have confirmed the regulatory role of HDAC6 in QC autophagy, but not in starvation-induced autophagy, through recognizing poly-ubiquitinated proteins and controlling the fusion process of autophagosomes and lysosomes $(3,47,57,58)$. HDAC6 is a multidomain protein including a BUZ-finger domain, which binds to polyubiquitin chains, and two deacetylase domains interrupted by a motif that binds to cytoplasmic dynein (36), which enable efficient recruitment and delivery of aggregates to autophagosomes via the microtubule cytoskeleton $(46,48)$. It has been shown to be involved in aggresome formation and the fusion of autophagosomes with lysosomes (31). Lee et al (3) demonstrated that HDAC6 is a central component of basal autophagy that targets protein aggregates and damaged mitochondria, and controls autophagosome maturation, which is essential for ubiquitin-selective QC autophagy (3). In the present study, the effect of hnRNPKknockdown on QC autophagy could be partially reverted by Tub A, an inhibitor of HDAC6 deacetylation activity. This highlights the role of HDAC6 in the acetylation of $\alpha$-tubulin in addition to its binding to polyubiquitin chains, further confirming the dual action of HDAC6 in the degradation of the ubiquitinated protein aggregates during QC autophagy.

hnRNPK, shuttling between the nucleus and cytoplasm, serves as a docking platform that integrates signal transduction pathways and regulates the expression of specific genes in the cell through the regulation of RNA metabolism and expression. It has been demonstrated to control the expression of tumor suppressor p53, which may have positive and negative regulatory roles in autophagy induction (59-62). However, the present study demonstrated that hnRNPK negatively regulated autophagy under normal nutrient conditions, and seemed to be dispensable for non-selective starvation- and rapamycin-induced autophagy, implying that the regulation of p53 activity during the autophagy processes is associated with a complex regulatory mechanism in which other factors may also participate. Despite its long-term argumentation toward the origin, phenotype, karyotype and tumorigenicity, the 293 cell line is one of the most commonly used cell models in cell biology studies due to its reliable growth and propensity for transfection. Therefore, the 293 cell line was used in the present study as a general cell model, without any particular purpose associated with the origin of the cell, for the study of cell autophagy that serves important physiological roles in a variety of cells (63-65).

To the best of our knowledge, our previous study was the first to describe and discuss the regulation of the $\alpha$-tubulin K40 acetylation by hnRNPK and its functional impact in multi-nucleated mature osteoclasts (30). The present study attempted to confirm a similar function of hnRNPK in the context of autophagy regulation in 293 cells, and to further investigate the underlying mechanism through the regulation of HDAC6 expression. The results of the present study demonstrated that hnRNPK negatively regulates the expression levels of HDAC6, but the precise underlying mechanism of this remains to be elucidated. Belonging to the hnRNP family, hnRNPK has been most extensively studied as a DNA/RNA binding protein regulating mRNA metabolisms, including the synthesis, maturation, trafficking, stability and translational modulation (10-13). The present study analyzed the mRNA expression levels of HDAC6 in the hnRNPK ${ }^{+-}$and wildtype 293 cells and demonstrated that the mRNA and protein expression of HDAC6 was increased in the hnRNPK ${ }^{+/}-2$ cells compared with the wild-type cells, suggesting that hnRNPK may regulate the synthesis or stability of HDAC6 mRNA rather than the translation. Further studies would aid in elucidating the precise mechanism of hnRNPK-mediated regulation of HDAC6 expression.

Taken together, the results of the present study indicated that hnRNPK serves a key role in the ubiquitin-selective QC autophagy by regulating the expression of HDAC6, which is required for successful autophagic clearance of protein aggregates and efficient fusion of autophagosomes with lysosomes, which contributes toward the elucidation of the components of the autophagic machinery.

\section{Acknowledgements}

The authors would like to thank members of the Key Laboratory of Functional Protein Research of Guangdong Higher Education Institutes for providing discussion and support throughout the present study.

\section{Funding}

The present study was supported by grants from the Guangdong Natural Science Foundation, Guangdong, China (grant nos. S2013030013315 and 2016A030313083) and the 
Science and Technology Program of Guangzhou, Guangdong, China (grant no. 201607010175).

\section{Availability of data and materials}

All datasets used and/or analyzed during the current study are available from the corresponding author on reasonable request.

\section{Authors' contributions}

LZ, LX and LL conceived and designed the experiments. LZ, MJ and ZT performed the experiments. LZ, LX, GX and LL analyzed the data. LX and LL wrote the main manuscript text, and LZ and LX prepared the figures. All authors read and approved the final manuscript.

\section{Ethics approval and consent to participate}

Not applicable.

\section{Patient consent for publication}

Not applicable.

\section{Competing interests}

The authors declare that they have no competing interests.

\section{References}

1. Mizushima N: Autophagy: Process and function. Genes Dev 21 2861-2873, 2007

2. Mizushima N, Levine B, Cuervo AM and Klionsky DJ: Autophagy fights disease through cellular self-digestion. Nature 451: 1069-1075, 2008.

3. Lee JY, Koga H, Kawaguchi Y, Tang W, Wong E, Gao YS, Pandey UB, Kaushik S, Tresse E, Lu J, et al: HDAC6 controls autophagosome maturation essential for ubiquitin-selective quality-control autophagy. EMBO J 29: 969-980, 2010.

4. Sun K, Deng W, Zhang S, Cai N, Jiao S, Song J and Wei L: Paradoxical roles of autophagy in different stages of tumorigenesis: Protector for normal or cancer cells. Cell Biosci 3: 35, 2013.

5. Rogov V, Dötsch V, Johansen T and Kirkin V: Interactions between autophagy receptors and ubiquitin-like proteins form the molecular basis for selective autophagy. Mol Cell 53: 167-178, 2014.

6. Pankiv S, Clausen TH, Lamark T, Brech A, Bruun JA, Outzen H, Øvervatn A, Bjørkøy G and Johansen T: p62/SQSTM1 binds directly to Atg8/LC3 to facilitate degradation of ubiquitinated protein aggregates by autophagy. J Biol Chem 282: 24131-24145, 2007.

7. Behrends C, Sowa ME, Gygi SP and Harper JW: Network organization of the human autophagy system. Nature 466: 68-76, 2010.

8. Galluzzi L, Pietrocola F, Levine B and Kroemer G: Metabolic control of autophagy. Cell 159: 1263-1276, 2014.

9. He C and Klionsky DJ: Regulation mechanisms and signaling pathways of autophagy. Annu Rev Genet 43: 67-93, 2009.

10. Michelotti EF, Tomonaga T, Krutzsch H and Levens D: Cellular nucleic acid binding protein regulates the CT element of the human c-myc protooncogene. J Biol Chem 270: 9494-9499, 1995.

11. Michelotti EF, Michelotti GA, Aronsohn AI and Levens D: Heterogeneous nuclear ribonucleoprotein $\mathrm{K}$ is a transcription factor. Mol Cell Biol 16: 2350-2360, 1996.

12. Miau LH, Chang CJ, Shen BJ, Tsai WH and Lee SC: Identification of heterogeneous nuclear ribonucleoprotein $\mathrm{K}$ (hnRNP K) as a repressor of C/EBPbeta-mediated gene activation. J Biol Chem 273: 10784-10791, 1998.
13. Chen LC, Chung IC, Hsueh C, Tsang NM, Chi LM, Liang Y, Chen CC, Wang LJ and Chang YS: The antiapoptotic protein, FLIP, is regulated by heterogeneous nuclear ribonucleoprotein $\mathrm{K}$ and correlates with poor overall survival of nasopharyngeal carcinoma patients. Cell Death Differ 17: 1463-1473, 2010.

14. Bomsztyk K, Denisenko O and Ostrowski J: hnRNP K: One protein multiple processes. BioEssays 26: 629-638, 2004.

15. Schullery DS, Ostrowski J, Denisenko ON, Stempka L, Shnyreva M, Suzuki H, Gschwendt M and Bomsztyk K: Regulated interaction of protein kinase Cdelta with the heterogeneous nuclear ribonucleoprotein K protein. J Biol Chem 274: 15101-15109, 1999.

16. Habelhah H, Shah K, Huang L, Ostareck-Lederer A, Burlingame AL, Shokat KM, Hentze MW and Ronai Z: ERK phosphorylation drives cytoplasmic accumulation of hnRNP-K and inhibition of mRNA translation. Nat Cell Biol 3: 325-330, 2001.

17. Ostareck-Lederer A, Ostareck DH, Cans C, Neubauer G, Bomsztyk K, Superti-Furga G and Hentze MW: c-Src-mediated phosphorylation of hnRNP K drives translational activation of specifically silenced mRNAs. Mol Cell Biol 22: 4535-4543, 2002.

18. Miyazaki T, Sanjay A, Neff L, Tanaka S, Horne WC and Baron R: Src kinase activity is essential for osteoclast function. J Biol Chem 279: 17660-17666, 2004.

19. Wolf D, Witte V, Clark P, Blume K, Lichtenheld MG and Baur AS: HIV Nef enhances Tat-mediated viral transcription through a hnRNP-K-nucleated signaling complex. Cell Host Microbe 4: 398-408, 2008.

20. Chang JW, Koike T and Iwashima M: hnRNP-K is a nuclear target of TCR-activated ERK and required for T-cell late activation. Int Immunol 21: 1351-1361, 2009.

21. Hutchins EJ and Szaro BG: c-Jun N-terminal kinase phosphorylation of heterogeneous nuclear ribonucleoprotein $\mathrm{K}$ regulates vertebrate axon outgrowth via a posttranscriptional mechanism. J Neurosci 33: 14666-14680, 2013.

22. Gao X, Feng J, He Y, Xu F, Fan X, Huang W, Xiong H, Liu Q, Liu W, Liu X, et al: hnRNPK inhibits GSK3 $\beta$ Ser9 phosphorylation, thereby stabilizing c-FLIP and contributes to TRAIL resistance in H1299 lung adenocarcinoma cells. Sci Rep 6: 22999, 2016.

23. Carpenter B, McKay M, Dundas SR, Lawrie LC, Telfer C and Murray GI: Heterogeneous nuclear ribonucleoprotein $\mathrm{K}$ is over expressed, aberrantly localised and is associated with poor prognosis in colorectal cancer. Br J Cancer 95: 921-927, 2006.

24. Barboro P, Repaci E, Rubagotti A, Salvi S, Boccardo S, Spina B, Truini M, Introini C, Puppo P, Ferrari N, et al: Heterogeneous nuclear ribonucleoprotein $\mathrm{K}$ : Altered pattern of expression associated with diagnosis and prognosis of prostate cancer. Br J Cancer 100: 1608-1616, 2009.

25. Matta A, Tripathi SC, DeSouza LV, Grigull J, Kaur J, Chauhan SS, Srivastava A, Thakar A, Shukla NK, Duggal R, et al: Heterogeneous ribonucleoprotein $\mathrm{K}$ is a marker of oral leukoplakia and correlates with poor prognosis of squamous cell carcinoma. Int J Cancer 125: 1398-1406, 2009.

26. Chen LC, Hsueh C, Tsang NM, Liang Y, Chang KP, Hao SP, Yu JS and Chang YS: Heterogeneous ribonucleoprotein K and thymidine phosphorylase are independent prognostic and therapeutic markers for nasopharyngeal carcinoma. Clin Cancer Res 14: 3807-3813, 2008

27. Gallardo M, Lee HJ, Zhang X, Bueso-Ramos C, Pageon LR, McArthur M, Multani A, Nazha A, Manshouri T, ParkerThornburg J, et al: hnRNP K is a haploinsufficient tumor suppressor that regulates proliferation and differentiation programs in hematologic nalignancies. Cancer Cell 28: 486-499, 2015.

28. Barboro P, Ferrari N and Balbi C: Emerging roles of heterogeneous nuclear ribonucleoprotein $\mathrm{K}$ ( $\mathrm{hnRNP} \mathrm{K})$ in cancer progression. Cancer Lett 352: 152-159, 2014.

29. Zhang J, Liu X, Lin Y, Li Y, Pan J, Zong S, Li Y and Zhou Y: HnRNP K contributes to drug resistance in acute myeloid leukemia through the regulation of autophagy. Exp Hematol 44: 850-856, 2016.

30. Fan X, Xiong H, Wei J, Gao X, Feng Y, Liu X, Zhang G, He QY, $\mathrm{Xu}$ J and Liu L: Cytoplasmic hnRNPK interacts with GSK3ß and is essential for the osteoclast differentiation. Sci Rep 5: 17732, 2015.

31. Köchl R, Hu XW, Chan EY and Tooze SA: Microtubules facilitate autophagosome formation and fusion of autophagosomes with endosomes. Traffic 7: 129-145, 2006. 
32. Xie R, Nguyen S, McKeehan WL and Liu L: Acetylated microtubules are required for fusion of autophagosomes with lysosomes. BMC Cell Biol 11: 89, 2010.

33. Al-Bassam J and Corbett KD: $\alpha$-Tubulin acetylation from the inside out. Proc Natl Acad Sci USA 109: 19515-19516, 2012.

34. Friedmann DR, Aguilar A, Fan J, Nachury MV and Marmorstein R: Structure of the $\alpha$-tubulin acetyltransferase, aTAT1, and implications for tubulin-specific acetylation. Proc Natl Acad Sci USA 109: 19655-19660, 2012.

35. Taschner M, Vetter M and Lorentzen E: Atomic resolution structure of human $\alpha$-tubulin acetyltransferase bound to acetylCoA. Proc Natl Acad Sci USA 109: 19649-19654, 2012.

36. Hubbert C, Guardiola A, Shao R, et al: HDAC6 is a microtubuleassociated deacetylase. Nature 417: 455-458, 2002.

37. Kim SH, Shanware NP, Bowler MJ and Tibbetts RS: Amyotrophic lateral sclerosis-associated proteins TDP-43 and FUS/TLS function in a common biochemical complex to co-regulate HDAC6 mRNA. J Biol Chem 285: 34097-34105, 2010.

38. Zhu Y, Qin Z, Gao J, Yang M, Qin Y, Shen T and Liu S: Vitamin D therapy in experimental allergic encephalomyelitis could be limited by opposing effects of sphingosine 1-phosphate and gelsolin dysregulation. Mol Neurobiol 50: 733-743, 2014.

39. Pan Z, Ding Q, Guo Q, Guo Y, Wu L, Wu L, Tang M, Yu H and Zhou F: MORC2, a novel oncogene, is upregulated in liver cancer and contributes to proliferation, metastasis and chemoresistance. Int J Oncol 53: 59-72, 2018.

40. Wyant GA, Abu-Remaileh M, Wolfson RL, Chen WW, Freinkman E, Danai LV, Vander Heiden MG and Sabatini DM: mTORC1 activator SLC38A9 is required to efflux essential amino acids from lysosomes and use protein as a nutrient. Cell 171: 642-654.e12, 2017.

41. Qian X, Li X, Cai Q, Zhang C, Yu Q, Jiang Y, Lee JH, Hawke D, Wang Y, Xia Y, et al: Phosphoglycerate kinase 1 phosphorylates Beclin1 to induce autophagy. Mol Cell 65: 917-931.e6, 2017.

42. Barrangou R, Birmingham A, Wiemann S, Beijersbergen RL, Hornung V and Smith A: Advances in CRISPR-Cas9 genome engineering: Lessons learned from RNA interference. Nucleic Acids Res 43: 3407-3419, 2015.

43. Hsu PD, Lander ES and Zhang F: Development and applications of CRISPR-Cas9 for genome engineering. Cell 157: 1262-1278, 2014.

44. Matsuyama A, Shimazu T, Sumida Y, Saito A, Yoshimatsu Y, Seigneurin-Berny D, Osada H, Komatsu Y, Nishino N, Khochbin $\mathrm{S}$, et al: In vivo destabilization of dynamic microtubules by HDAC6-mediated deacetylation. EMBO J 21 6820-6831, 2002.

45. Kalebic N, Sorrentino S, Perlas E, Bolasco G, Martinez C and Heppenstall PA: $\alpha$ TAT1 is the major $\alpha$-tubulin acetyltransferase in mice. Nat Commun 4: 1962, 2013.

46. Iwata A, Riley BE, Johnston JA and Kopito RR: HDAC6 and microtubules are required for autophagic degradation of aggregated huntingtin. J Biol Chem 280: 40282-40292, 2005.

47. Ouyang H, Ali YO, Ravichandran M, Dong A, Qiu W, MacKenzie F, Dhe-Paganon S, Arrowsmith $\mathrm{CH}$ and Zhai RG: Protein aggregates are recruited to aggresome by histone deacetylase 6 via unanchored ubiquitin $\mathrm{C}$ termini. J Biol Chem 287: 2317-2327, 2012.

48. Hook SS, Orian A, Cowley SM and Eisenman RN: Histone deacetylase 6 binds polyubiquitin through its zinc finger (PAZ domain) and copurifies with deubiquitinating enzymes. Proc Natl Acad Sci USA 99: 13425-13430, 2002.
49. Mizushima N: The pleiotropic role of autophagy: From protein metabolism to bactericide. Cell Death Differ 12 (Suppl 2): 1535-1541, 2005.

50. Monastyrska I, Rieter E, Klionsky DJ and Reggiori F: Multiple roles of the cytoskeleton in autophagy. Biol Rev Camb Philos Soc 84: 431-448, 2009.

51. Kimura S, Noda T and Yoshimori T: Dynein-dependent movement of autophagosomes mediates efficient encounters with lysosomes. Cell Struct Funct 33: 109-122, 2008.

52. Jahreiss L, Menzies FM and Rubinsztein DC: The itinerary of autophagosomes: From peripheral formation to kiss-and-run fusion with lysosomes. Traffic 9: 574-587, 2008.

53. Reunanen H, Marttinen M and Hirsimäki P: Effects of griseofulvin and nocodazole on the accumulation of autophagic vacuoles in Ehrlich ascites tumor cells. Exp Mol Pathol 48: 97-102, 1988.

54. Mackeh R, Perdiz D, Lorin S, Codogno P and Poüs C: Autophagy and microtubules - new story, old players. J Cell Sci 126: 1071-1080, 2013.

55. Lamark T and Johansen T: Aggrephagy: Selective disposal of protein aggregates by macroautophagy. Int J Cell Biol 2012: $736905,2012$.

56. Hubbert C, Guardiola A, Shao R, Kawaguchi Y, Ito A, Nixon A, Yoshida M, Wang XF and Yao TP: HDAC6 is a microtubuleassociated deacetylase. Nature 417: 455-458, 2002.

57. Lin TW, Chen MT, Lin LT, Huang PI, Lo WL, Yang YP, Lu KH, Chen YW, Chiou SH and Wu CW: TDP-43/HDAC6 axis promoted tumor progression and regulated nutrient deprivationinduced autophagy in glioblastoma. Oncotarget 8: 56612-56625, 2017.

58. Pandey UB, Nie Z, Batlevi Y, McCray BA, Ritson GP, Nedelsky NB, Schwartz SL, DiProspero NA, Knight MA, Schuldiner O, et al: HDAC6 rescues neurodegeneration and provides an essential link between autophagy and the UPS Nature 447: 859-863, 2007.

59. Galindo-Moreno M, Giráldez S, Sáez C, Japón MA, Tortolero M and Romero F: Both p62/SQSTM1-HDAC6-dependent autophagy and the aggresome pathway mediate CDK1 degradation in human breast cancer. Sci Rep 7: 10078, 2017.

60. Blasius M and Bartek J: ATM targets hnRNPK to control p53. Cell Cycle 12: 1162-1163, 2013.

61. Zong WX and Moll U: p53 in autophagy control. Cell Cycle 7: 2947-2948, 2008.

62. Balaburski GM, Hontz RD and Murphy ME: p53 and ARF: Unexpected players in autophagy. Trends Cell Biol 20: 363-369, 2010.

63. Yang Z and Klionsky DJ: Mammalian autophagy: Core molecular machinery and signaling regulation. Curr Opin Cell Biol 22: 124-131, 2010.

64. Li D, Zhao K, Yang X, Xiao X and Tang S: TCS2 increases olaquindox-induced apoptosis by upregulation of ROS production and downregulation of autophagy in HEK293 cells. Molecules 22: 595, 2017.

65. Stepanenko AA and Dmitrenko VV: HEK293 in cell biology and cancer research: Phenotype, karyotype, tumorigenicity, and stress-induced genome-phenotype evolution. Gene 569: 182-190, 2015. 